\title{
Deformation and Transitions at Interfaces and Grain Boundaries
}

\author{
SARYU FENSIN, ${ }^{1,6}$ DOUG SPEAROT, ${ }^{2}$ TOM BIELER, ${ }^{3}$ \\ SHEN DILLON, ${ }^{4}$ and JIAN LUO ${ }^{5}$
}

1.-Los Alamos National Laboratory, Los Alamos, NM, USA. 2.-University of Florida, Gainesville, FL, USA. 3.-Michigan State University, East Lansing, MI, USA. 4.-University of Illinois, Champaign, IL, USA. 5.-University of California, San Diego, La Jolla, CA, USA. 6.-e-mail: saryuj@lanl.gov

Materials form the backbone of every major invention in the history of humankind. For example, nuclear reactors or space shuttles would not exist without materials that can withstand extreme temperatures and radiation environments. The development of a new application or the attainment of a new goal is generally not limited by the technology itself but by the availability of a material with appropriate properties that can function under specific conditions and environments. For example, lightweight cars are needed to increase fuel efficiency and to decrease our dependence on fossil fuels. However, this goal cannot be achieved until we have a material that is not only lightweight but also has the strength of traditional materials, such as high-strength steel. There is an abundance of examples including future applications that cannot yet be functional due to the lack of appropriate materials. Thus, it is indispensable to not only design new materials but also to optimize the utilization of current materials. Even when guided by established principles, our current approach involves much trial and error, which makes the development of new materials slow and expensive. Despite all the progress in materials science, we still cannot accurately predict material strength and ductility properties even in pure FCC ductile metals, especially under extreme conditions.

In order to design materials to withstand extreme environments - either thermal, mechanical, or radiation-it is important to understand the factors that ultimately control material properties. In fact, this area has been extensively researched in the past decades and it is accepted that it is the material microstructure-chemistry, grain size, dislocation density, texture, to list a few-that control material

Saryu Fensin, Doug Spearot, Tom Bieler, Shen Dillon, and Jian Luo, guest editors for the Mechanical Behavior of Materials Committee of the TMS Structural Materials Division (SMD), coordinated the topic Deformation and Transitions at Grain Boundaries in this issue. properties. Some of these microstructural characteristics have been exploited since the beginning of time to increase material strength, a prime example being alloying. Other more recent attempts have focused on reducing grain size to generate materials with enhanced strength and ductility without altering the chemistry. This increase in strength has been described by the Hall-Petch equation, where grain boundaries act as barriers to dislocation motion. However, if the grain size is further reduced below $100 \mathrm{~nm}$, there is a precipitous increase in material properties that cannot simply be explained by Hall-Petch strengthening. A plethora of research on nanocrystalline materials shows that the cause of enhanced properties at small grain sizes is due to varying mechanisms of plastic deformation specifically associated with grain boundaries, in comparison to conventional polycrystalline materials. In fact, the last couple of decades have focused on not only understanding how grain boundaries increase strength in nanocrystalline materials, especially when coupled with chemistry, but have also provided new insights into grain boundary engineering.

Grain boundaries are microscopic defects and as a result it is difficult to perform in situ experiments to observe mechanisms that lead to heterogeneous properties as a function of boundary type. For example, why are certain boundaries more resistant to corrosion and radiation damage? Hence, it is critical to couple experiments with atomistic modeling to understand the origins of these special properties associated with specific boundary structures. Grain boundary engineering is an area of research that focuses on improving material properties by controlling the type of boundaries present in a material. It takes advantage of the fact that not all grain boundaries are equal in terms of their ability to affect properties. For example, certain "special" boundaries in metals have been shown to have a higher resistance to corrosion, possess higher strength, and even help mitigate damage from a 
radiation cascade. This approach, although interesting, relies on the fact that we understand "why" such specific relations between properties and specific boundary structures exist. Previous research has shown that grain boundaries are nucleation sites for dislocations, twins, and phase transformations. Deformation at the microstructural scale is heterogeneous, due to differences in elastic properties and deformation resistance of specific grain boundaries with respect to an externally applied stress. This leads to differences in strain accumulation, causes structural changes and transformations within the boundary, and generates gradients in neighboring grains. Due to these effects, the local stress and strain tensors can differ significantly from globally imposed stress states. However, we cannot predict the evolution of local stress tensors as a function of boundary type. The ability to confidently predict local stress states is key to the material design paradigm that could take advantage of a combination of enhanced properties of nanocrystals coupled with grain boundary engineering. In this issue, we have compiled both experimental and modeling papers that not only investigate how interfaces affect properties but also design approaches to enhance material properties under extreme conditions.

The following list summarizes the papers being published under the topic of "Deformation and Transitions at Grain Boundaries." To download any of the papers, follow the URL http://link.sprin ger.com/journal/11837/71/4/page/ 1 to the table of contents page for the April 2019 issue (vol. 71, no. $4)$.

- "Structures and Mechanical Properties of $\mathrm{Al} /$ $\mathrm{Al}_{2} \mathrm{Cu}$ Interfaces" by Guisen Liu, Mingyu Gong, Dongyue Xie, and Jian Wang.

- "Atomistic Study of the Effect of Magnesium Dopants on the Strength of Nanocrystalline Aluminum" by Amirreza Kazemi and Shengfeng Yang.

- "Interface Facilitated Reorientation of $\mathrm{Mg}$ Nanolayers in Mg-Nb Nanolaminates" by Youxing Chen, Mingyu Gong, Shuai Shao, Nathan Mara, and Jian Wang.

- "In Situ High-Cycle Fatigue Reveals the Importance of Grain Boundary Structure in Nanocrystalline Cu-Zr" by Jennifer D. Schuler, Christopher M. Barr, Nathan M. Heckman, Guild Copeland, Brad L. Boyce, Khalid Hattar, and Timothy J. Rupert.

- "Interplay Between Grain Boundaries and Radiation Damage" by Christopher M. Barr, Osman El-Atwani, Djamel Kaoumi, and Khalid Hattar.

Publisher's Note Springer Nature remains neutral with regard to jurisdictional claims in published maps and institutional affiliations. 